\title{
A blind search for a common signal in gravitational wave detectors
}

\section{Hao Liu, ${ }^{a, b}$ James Creswell, ${ }^{a}$ Sebastian von Hausegger, ${ }^{a}$ Andrew D. Jackson ${ }^{c}$ and Pavel Naselsky ${ }^{a}$}

\author{
${ }^{a}$ The Niels Bohr Institute \& Discovery Center, Blegdamsvej 17, DK-2100 Copenhagen, Denmark \\ ${ }^{b}$ Key laboratory of Particle and Astrophysics, Institute of High Energy Physics, CAS, 19B YuQuan \\ Road, Beijing, China \\ ${ }^{c}$ The Niels Bohr International Academy, Blegdamsvej 17, DK-2100 Copenhagen, Denmark \\ E-mail: liuhao@nbi.dk,dgz764@alumni.ku.dk, s.vonhausegger@nbi.dk, \\ jackson@nbi.dk, naselsky@nbi.dk
}

\begin{abstract}
We propose a blind, template-free method for the extraction of a common signal between the Hanford and Livingston detectors and apply it especially to the GW150914 event. We construct a log-likelihood method that maximizes the cross-correlation between each detector and the common signal and minimizes the cross-correlation between the residuals. The reliability of this method is tested using simulations with an injected common signal. Finally, our method is used to assess the quality of theoretical gravitational wave templates for GW150914.
\end{abstract}

Keywords: gravitational waves / experiments, gravitational waves / sources 


\section{Contents}

1 Introduction 1

2 Definitions and likelihood approach 2

$\begin{array}{llr}2.1 \text { Cross-correlations and residuals } & 2\end{array}$

$\begin{array}{lll}2.2 & \text { The likelihood approach } & 3\end{array}$

3 The search algorithm applied to Hanford and Livingston data 4

3.1 Pre-processing of Hanford and Livingston data 4

$\begin{array}{ll}3.2 & \text { The search algorithm } \\ \end{array}$

4 Oscillations in the likelihood function $\quad 6$

5 Test by simulation $\quad 7$

6 Testing the residuals $\quad 8$

6.1 The residual correlation $\quad 8$

6.2 Determination of the threshold 10

7 Goodness of the GW template as an estimator of the common signal 11

8 Discussion $\quad 14$

\section{Introduction}

To date five gravitational wave (GW) events from binary black hole mergers have been announced by the LIGO/Virgo collaboration [1-5], among which GW150914 is the most statistically significant. This event provides a unique opportunity for the investigation of both templates and noise [6]. In spite of the extensive discussion in the literature devoted to the exploitation of the physical consequences of this observation, remarkably few papers have addressed the morphological properties of the signal and the significance of its detection [7, 8]. Unlike the other LIGO events (GW151226, GW170104, GW170814 and the most recent GW170817), GW150914 can be identified in the time ordered data stream without the use of the templates [8]. In this case, the use of templates can be restricted to the determination of the masses, spins and distance to the presumed black hole binary system. To the extent that assumptions regarding the nature of the event are correct and the properties of the noise are well understood, these physical parameters can be estimated using maximum likelihood methods. However, searches for a common signal using methods that are based solely on the use of templates have the potential danger of misidentifying transients and/or systematic effects as part of the desired signal. At the very least, preconceptions can introduce a bias in the estimation of the inferred parameters. Such limitations on the reliability of template-based signal extraction methods emphasize the desirability of template-free methods.

In this paper we propose such a template-free method for determining the best common signal in the Hanford and Livingston detectors. While nothing prevents application of this method to any event with a sufficiently high signal-to-noise ratio, in this paper we focus on the $0.2 \mathrm{~s}$ of the GW150914 event. The physical basis of the method is that there exists a common signal in the LIGO Hanford and Livingston detectors. The presence of this signal, $A(t)$, in each of the two detectors is characterised by 
the cross-correlation coefficients $C_{A D}$, giving the residuals $R_{D}(t)=D(t)-C_{A D} \cdot A(t)$, where $D(t)$ is the strain data for Hanford $(D=H)$ or Livingston $(D=L)$. Our objective is to find $A(t)$ such that the cross-correlation between $A(t)$ and each of the two data streams $D(t)$ is maximized, while the cross-correlation between $R_{H}(t)$ and $R_{L}(t)$ is minimized. For this purpose, a maximum likelihood approach for the cross-correlation functions is constructed by means of their Fisher transforms [9], in combination with a random search for the optimal solution $A(t)$.

An important feature of our approach is that, unlike template fitting, we obtain a family of optimal solutions, $A_{i}(t)$, that differ from one another because of chance correlations between $A_{i}(t)$ and each of $R_{H, i}(t)$ and $R_{L, i}(t)$, and between $R_{H, i}(t)$ and $R_{L, i}(t)$. These chance correlations are an unavoidable part of the optimization technique, and they reflect the intrinsic statistical properties of the problem.

Other template-free methods have been designed for detection and identification of gravitational waves, such as the oLIB method [10], the X-pipeline method [11], and the BayesWave method [16, 17]. Our method is intended to be used as a follow-up to extract the common signal from detection candidates. Unlike other approaches, it is completely blind and does not explicitly depend on the amplitude.

Also note that in this work, we use the Hanford detector as the default projection (see eq. 3.1), so all figures will be suitable for comparison with Hanford data.

The outline of the paper is as follows. In section 2 we present definitions and construct the likelihood function. In section 3 we introduce the necessary modifications of the data streams to apply the method to LIGO data and describe the algorithm. The non-uniqueness of the solution due to chance correlations is discussed in section 4 including first results, followed by testing for the reliability of the method by the use of simulations in section 5 . We then investigate correlations between the residuals in section 6 , and in section 7 we evaluate binary black hole merger templates for their goodness of fit to the common signal.

\section{Definitions and likelihood approach}

\subsection{Cross-correlations and residuals}

Assume two data sets $X(t)$ and $Y(t)$ (e.g. strain data from two independent detectors) which contain a common signal $A(t)$. For convenience, we assume that in the time range of interest $X, Y$, and $A$ have their average value shifted to zero and their variance normalized to unity. With this simplification the cross-covariance and the Pearson cross-correlation coefficient of two vectors of size $N$ are equal. ${ }^{1}$

$$
S_{X Y}=\frac{1}{N-1} \sum_{i=1}^{N} X_{i} \cdot Y_{i} \quad C_{X Y}=\frac{S_{X Y}}{\sqrt{S_{X X} S_{Y Y}}}=S_{X Y} .
$$

We calculate the residuals according to linear regression of $A(t)$ against $X(t)$ or $Y(t)$ :

$$
\begin{gathered}
R_{X}=X-A \cdot \frac{S_{A X}}{S_{A A}}=X-A \cdot S_{A X}, \\
R_{Y}=Y-A \cdot \frac{S_{A Y}}{S_{A A}}=Y-A \cdot S_{A Y},
\end{gathered}
$$

where in the last steps we have again made use of the signals having been scaled to unit variance. It is important to note that, by construction, the correlations of both $R_{X}$ and $R_{Y}$ with $A$ are zero. Since

\footnotetext{
${ }^{1}$ Throughout this paper we will change between the notations $X=X(t)$ and $X_{i}=X\left(t_{i}\right)$ at convenience.
} 
it might be expected that the residuals are defined as the difference between the data and the best common signal, the factors of $S_{A X}$ and $S_{A Y}$ in eqs. 2.2 may seem surprising. This expectation can be fulfilled by a simple rescaling of $A$ which will have no influence on the results of the following procedure which is based solely on morphology. Note, too, that the amplitude of template (i.e., the analogue of the best common signal) is also freely adjustable in LIGO's analysis.

The criterion for our blind estimation of $A(t)$ now is to maximize $C_{A X}$ and $C_{A Y}$ while simultaneously minimizing the cross-correlation between the residuals, $C_{R_{X} R_{Y}}$. These can be obtained straightforwardly from eqs. 2.1. Note that in $C_{R_{X} R_{Y}}$ the residuals $R_{X}$ and $R_{Y}$ are not automatically normalized:

$$
C_{R_{X} R_{Y}}=\frac{S_{R_{X} R_{Y}}}{\sqrt{S_{R_{X} R_{X}} \cdot S_{R_{Y} R_{Y}}}}=\frac{S_{X Y}-S_{A X} \cdot S_{A Y}}{\sqrt{\left(1-S_{A X}^{2}\right)\left(1-S_{A Y}^{2}\right)}} .
$$

\subsection{The likelihood approach}

In order to make statements about the likelihood of the resulting correlations, we need to estimate their distribution function. For simplicity, we obtain approximate Gaussianity by using the Fisher transformation [9]:

$$
Z_{X Y}=\frac{1}{2} \log \left(\frac{1+C_{X Y}}{1-C_{X Y}}\right)
$$

where $C$ is the Pearson cross-correlation coefficient from the previous section.

We now define the log-likelihood function that we will use in the remainder of this work:

$$
\log (L)=\left(Z_{A X}-E_{A X}\right)^{2}+\left(Z_{A Y}-E_{A Y}\right)^{2}-k\left(Z_{R_{X} R_{Y}}-E_{R_{X} R_{Y}}\right)^{2},
$$

where $E$ is the expectation of $Z$ and $k$ is a weighting factor, introduced to adjust the relative importance of the low correlation in the residuals over those of the data sets $X$ and $Y$ with $A$. A larger value of $k$ places greater emphasis on suppressing the residual correlation. Empirically, we find that setting $k=8$ works well, i.e. the correlations of the residuals are low and those of the common signal with the data are high, so we will keep this choice throughout this work. ${ }^{2}$

As mentioned previously, we expect no correlation between $R_{X}$ and $R_{Y}$, so we can set $E_{R_{X} R_{Y}}=0$ as the part of the initial null hypothesis that we wish to accept. In the search for a common signal, $A$, we similarly define the null hypothesis that $A$ is uncorrelated with each of $X$ and $Y$, and therefore fix $E_{A X}=E_{A Y}=0$. However, we wish to reject this null hypothesis, which leads us choose the sign of the first two terms in eq. 2.5 to be opposite to that of the third term. Given these considerations, eq. 2.5 reduces to ${ }^{3}$ :

$$
\log (L)=Z_{A X}^{2}+Z_{A Y}^{2}-k Z_{R_{X} R_{Y}}^{2} .
$$

A search based on eq. 2.6 also allows for an unwanted, peculiar solution

$$
A(t)=X(t)+O(t)
$$

where $|O(t)| \ll|X(t)|$ and is uncorrelated with $R_{Y}(t)$. In this case, $Z_{A X}$ will be very large, while $Z_{R_{X} R_{Y}}$ will still be small, resulting in an unreasonably large value of $\log (L)$. The common signal solution will tend to be close to either Hanford or Livingston data, leaving almost negligible residuals in the corresponding detector. This will result in an inhomogeneity of residuals that is inconsistent

\footnotetext{
${ }^{2}$ As the amplitude of the residuals can be small this makes no statement about the rough shape of the common signal. We offer a brief comparison of different choices of $k$ in section 5 .

${ }^{3}$ It is straightforward to generalize to three or more detectors by adding the corresponding terms to this equation.
} 
with the properties of noise observed outside the GW150914 domain. Therefore, for any solution $A$, with $C_{A X}$ or $C_{A Y}>0.9,{ }^{4}$ we reset $C_{A X}$ and/or $C_{A Y}$ to 0.9 to reduce preference of this type of solution. Note that this readjustment only affects the likelihood; it does not change the estimation or the data. Thus the actual values for $C_{A X}$ and/or $C_{A Y}$ can be higher than this limit, but the increase in correlation will not make the associated common signal more preferable in the search.

It should be noted that, since we base our search on computations of Pearson's cross-correlation coefficients, any amplitude difference between the two signals $X(t)$ and $Y(t)$ (as could be the case for signals from two gravitational wave detectors) is irrelevant. The search is expected to extract the most probable common signal regardless of the observed relative amplitude.

The criterion presented here for defining the best common signal is not unique. For example, the correlators $C_{A R_{X}}$ and $C_{A R_{Y}}$ are not expected to be strictly zero as a consequence of chance correlations. It would thus also be possible to replace the constraints imposed in eqs. 2.2 with additional terms proportional to $Z_{A R_{X}}^{2}$ and $Z_{A R_{Y}}^{2}$ in eq. 2.6. In situations where the signal-to-noise ratio in $X$ and $Y$ differ significantly, it could be useful to adjust the relative weight of the first two terms in eqs. 2.5 and 2.6 or to make independent adjustments of the upper limits for $C_{A X}$ and $C_{A Y}$. Any of these changes would introduce additional physically meaningful parameters in the likelihood function. Their presence would complicate the discussion but would not alter the principle of the method proposed here.

\section{The search algorithm applied to Hanford and Livingston data}

\subsection{Pre-processing of Hanford and Livingston data}

In order to apply the ideas above to the strain data $H(t)$ and $L(t)$ from the LIGO Hanford and Livingston detectors, respectively, it is necessary to pre-process the data by band-passing the data to the frequency range of interest ${ }^{5}$ and subsequently correcting for the phase difference $\Delta$ and the time lag $\tau{ }^{6}$ This can be done by modifying the Livingston Fourier coefficients $L(\omega)$ into

$$
\tilde{L}(\omega)=\alpha L(\omega) e^{i(\Delta+\omega \tau)}
$$

where $\Delta$ and $\tau$ are chosen to "match" $\tilde{L}(t)$ with $H(t)$. The constant $\alpha$ represents the difference in the detector efficiency, which is of no relevance in our method.

As an example, the LIGO Livingston template ${ }^{7}$ presented in figure 1 of [1] can be converted to the Hanford template by eq. 3.1 with $\alpha=1.23, \tau=6.96 \mathrm{~ms}$, and $\Delta=2.72$, as shown in figure 1 . However, by matching the real Livingston data to Hanford's, we find $\tau=7.08 \mathrm{~ms}$ and $\Delta=2.86$, only marginally different than the values for the templates. In this work, we will use $\tau=7.08 \mathrm{~ms}$ and $\Delta=2.86$ for pre-processing; the final result is insensitive to such small differences.

\footnotetext{
${ }^{4}$ Eq. 2.5 is a standard definition of the log-likelihood function that requires 6 priors ( 3 expectations and corresponding RMS values). Although empirical priors are widely used in likelihood analysis, following the discussions in Sec. 2.2, we have reduced the number of empirical priors from 6 to 2 . One is $k$, and the other is the threshold, and both are tested to ensure that the results are not very sensitive to them (see section 5 and section 6.2). The threshold also ensures that the integrated probability/likelihood is finite, which is required by self-consistency.

${ }^{5} \mathrm{We}$ frequency filter the data via a fourth-order (back-and- forth) Butterworth filter which passes frequencies between 35 and $350 \mathrm{~Hz}$ and additionally notch filter the narrow resonances, in accordance with ref. [1].

${ }^{6}$ See also the LIGO tutorial at http://Iosc. Iigo.org/s/events/GW150914/LOSC_Event_tutorial_ GW150914.html.

${ }^{7}$ Throughout this work we will refer to this template as the "GW150914 template". This is not LIGO's best-fit template, however recent work [18] has shown that the filtered templates are remarkably similar for a wide range of black hole parameters. The effect of using templates based on different parameters is studied in section 7.
} 


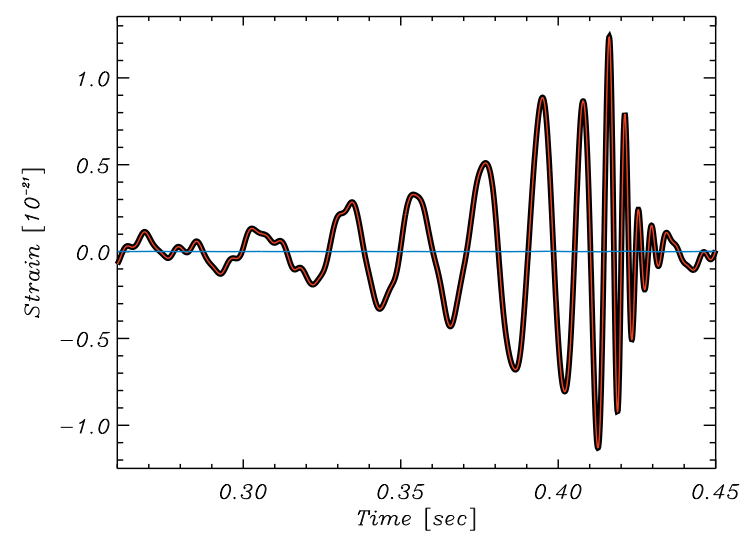

Figure 1. Matching the released Livingston template (red) to the Hanford template (black) by eq. 3.1 with $\alpha=1.23, \tau=6.96 \mathrm{~ms}$, and $\Delta=2.72$. Their difference after matching is shown in blue. Their cross correlation before matching is about 0.41 ; after matching it is greater than 0.99 .

The matching described and illustrated above is central to much of LIGO/Virgo's matched filter technique, including the primary results regarding GW150914 [19, 20]. However, the validity of the use of matched filters requires several assumptions regarding the underlying gravitational wave signal that are not true in general. In particular, the gravitational waves emitted by a binary system that is precessing (i.e. with component spins not aligned with the orbit axis) or a binary system with non-zero orbital eccentricity will induce strains in two detectors that are not equivalent after matching. Similarly, higher-order modes in the gravitational waves will result in differences between the strain data in two detectors that cannot be absorbed by the modifications described in eq. 3.1. In the presence of these complications, a common signal, $A(t)$, between the processed Hanford and Livingston strain data only exists approximately.

Furthermore, it is possible that there exist "foreground" components that introduce correlations in the Hanford and Livingston detectors that cannot be captured by a single common signal. For a qualitative exposition see the discussion in section 6.

\subsection{The search algorithm}

With the pre-processed data and the log-likelihood function presented in eq. 2.6, it is possible to run a point-by-point search for the best common signal. The algorithm proceeds iteratively as follows: we start with an initial guess of $A(t)$ which is simply random white noise. One after another, each data point is then moved by a small step $d(t)$ to produce a new signal,

$$
A^{\prime}(t)=A(t)+d(t) \cdot \delta\left(t, t_{i}\right),
$$

where $\delta\left(t, t_{i}\right)$ is a discrete Dirac delta function.

If $d(t)$ is sufficiently small, the change in the log-likelihood function will be linear. Thus if the likelihood function increases, we accept $+d(t)$ as the movement direction, otherwise we move the point in the opposite direction, $-d(t)$. This procedure is performed and recorded for each point independently. However, all changes are applied only after all directions have been determined, i.e. we ignore second- and higher-order partial derivatives like $\partial^{2} L / \partial A_{i} \partial A_{j}$ within one iteration. The updated estimation of $A(t)$ is then used for the next iterative step. 
Note that all analysis in this work can be done equivalently in either the time or frequency domain, where we have better control of the degrees of freedom (DOF). Since the data to be used is band-passed, we prefer to work in the frequency domain. However, the final results will be presented in the time domain for ease of understanding. Note, too, that at every iteration we correct the signals to obey the zero mean and unit variance conditions mentioned in section 2.1.

\section{Oscillations in the likelihood function}

Due to chance correlations between the residual and the real common signal, the result that gives the maximum likelihood is seldom precisely equal to the real common signal. When the iterative steps bring us sufficiently close to the true solution (or a local maximum), there will be a tendency for the estimations to oscillate. This is the case in our work. Since we start from a random white noise initial guess, the log-likelihood function will increase monotonically at the initial stages of the search, as shown in the left panel of figure 2. However, when the RMS difference between the "current" estimation and the real common signal is comparable to the residual noise, the likelihood function will start to oscillate, as shown by the right panel of figure 2 .
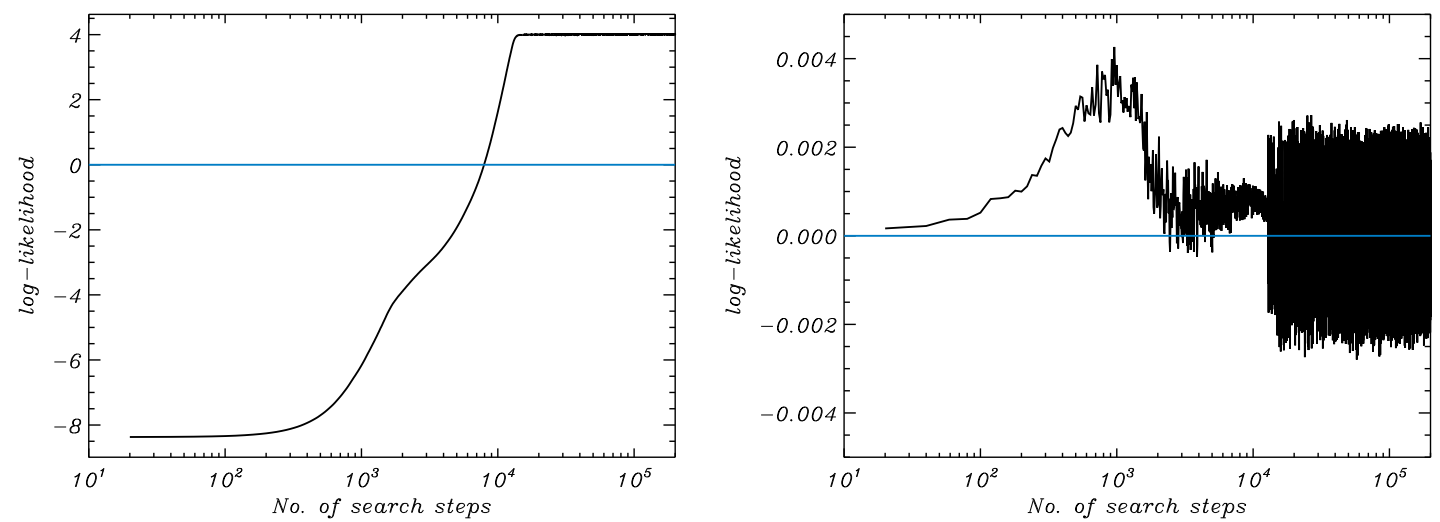

Figure 2. Left panel: the log-likelihood as a function of the iterative steps. Right panel: the oscillation of the $\log$-likelihood function, presented as $\log \left(L_{i+1}\right)-\log \left(L_{i}\right)$ (positive values stand for an increase in the likelihood.)

Therefore, in order to get a reasonable estimation of the common signal we choose the later half of the iterative steps $\left(10^{5}\right.$ steps, entirely in the oscillation region). Their average is the final estimation of one run $^{8}$ as shown in figure 3. Potentially, the fluctuation range for each point can also be given by the oscillation region; however, for two reasons, we prefer not to do this:

1. As mentioned above, the number of DOF in the frequency domain is much smaller than the number of points in the time domain. Thus, a point-to-point fluctuation range in the time domain does not reflect the actual fluctuation range which is more tightly constrained.

2. The fluctuation range for a single run is also related to the size of the iterative step, and its meaning is only relative.

\footnotetext{
${ }^{8}$ One run means running the blind search once, with one initial guess, and many iterative steps following the initial guess.
} 
For these reasons, we choose to use only the average solution in the oscillation region for a single run. To give an illustrative range of fluctuations, we repeat the procedure 100 times, starting each time with a different random initial guess. This will yield a range of fluctuations due to the existence of multiple local maxima, see figure 4. In this plot, we can see that the LIGO GW150914 template is not confined to the minimal-maximal uncertainty range, indicating that this template may not provide a reliable estimation of the common signal between Hanford and Livingston. However, we emphasize that figure 4 is only an illustration; an improved measure of the quality of the GW150914 template as an estimator of the true common signal will be given in section 7 .

The yellow band shown in figure 4 is important for two reasons. First, it is clear that this band provides a useful measure of the uncertainties associated with our determination of the best common signal and that this measure could be improved by increasing the number of trials. Second, the width of the yellow band would increase if the signal-to-noise ratio were smaller, and it would ultimately tell us that the signal is too weak to permit the blind extraction of a meaningful common signal. The existence of such an automatic warning of unreliability is an important feature of the present method not shared by template-based analyses.
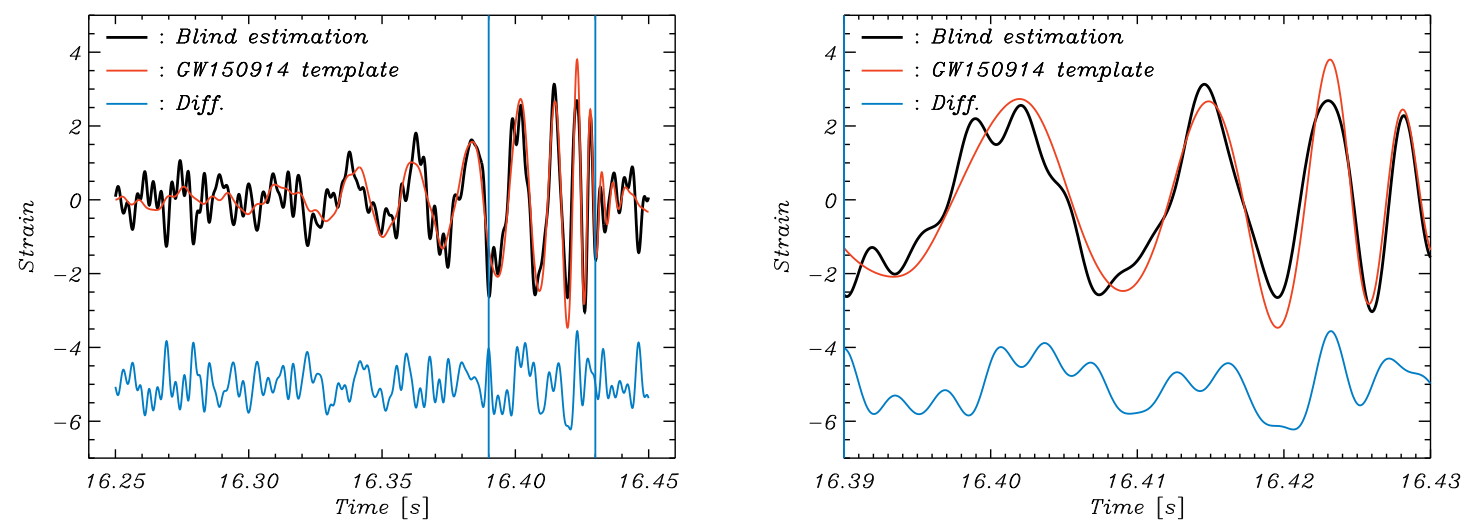

Figure 3. Our blind estimation (black) compared with the GW150914 template (red). Left panel: the whole $0.2 \mathrm{~s}$ range. Right panel: only the $0.39-0.43 \mathrm{~s}$ range (marked by the vertical lines in the left panel). The blind estimation denotes the average over all solutions in the second half of a single run $\left(2 \times 10^{5}\right.$ steps $)$, as described in the text.

\section{Test by simulation}

We have suggested a general method for obtaining the best common signal, applied it to the data for GW150914, and offered a rough impression of the associated uncertainties. We will now perform a simple test of our blind estimation method by using simulated data, where we have injected a known common signal into a noise background, as follows:

$$
\begin{aligned}
H^{\prime}(t) & =N_{H}(t)+A^{*}(t) \\
L^{\prime}(t) & =N_{L}(t)+A^{*}(t),
\end{aligned}
$$

where $A^{*}(t)$ is the known common signal, and $N_{H}(t), N_{L}(t)$ are noise backgrounds. The estimate of the common signal $A(t) \approx A^{*}(t)$ is obtained by the same algorithm as presented in section 3.2.

Trials indicate that the method extracts the injected signal equally well, regardless of the precise shape of the injected signal (GW150914 template or the common signal extracted above), as well as 


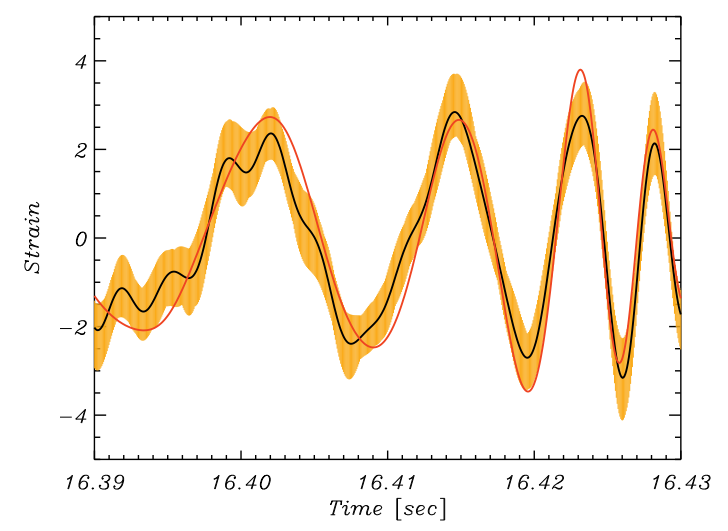

Figure 4. The minimal to maximal fluctuation range obtained for 100 runs (yellow band) and their average (black). Also shown is the GW150914 template for comparison (red). Note that the yellow range shown here is mainly for illustration, as described in the text.

of the noise model chosen (random white noise or real detector noise). The largest effect - although still marginal - is obtained when varying the initial guess. We therefore present results from injecting the common signal obtained above (and shown in figure 3) into real detector noise taken from detector data at least 3 seconds away from the GW-event. We run the search 100 times, and a different segment of detector noise is chosen for each run. The initial guess is varied, first setting it equal to the input signal, $A^{*}$, and, for comparison, equal to random white noise. ${ }^{9}$ figure 5 shows that virtually identical solutions are obtained either way. The fact that the solution always appears to be consistent with the known injected signal suggests that our method provides an unbiased estimate of the best common signal. ${ }^{10}$

We have also performed an additional test of the influence of the parameter $k$ in eq. 2.6 in which we set $k=0,2,4,8$. The resulting solutions are shown in figure 6 , from which we can see that the extracted signals are not very sensitive to the value of $k$. However, small differences in the template can lead to significant differences in the residual, which can be seen in the right panel of figure 6 .

\section{Testing the residuals}

\subsection{The residual correlation}

One of the defining properties of this method is the suppression of correlations in the residuals. After subtraction of the best common signal, we find a cross-correlation between the residuals in Hanford and Livingston of 0.13 in the $0.2 \mathrm{~s}$ time window. For comparison, the cross-correlation in the residuals after subtraction of the GW150914 template is 0.15. This value describes the correlation for the full region of investigation (in this case $0.2 \mathrm{~s}$ long). It is also relevant to consider this correlation on shorter time scales (see [6]). We can check the size of the correlations of residuals by calculating a running window correlation between the Hanford and Livingston residuals in each of the 100 runs

\footnotetext{
${ }^{9}$ In addition, new white noise is generated for each run with a random initial guess.

${ }^{10}$ Finally, it is interesting that we find a smaller fluctuation range in figure 5 (with real detector noise from outside the range of GW150914) than in figure 4 (with real detector noise from the time of GW150914), which suggests the residual noise associated with GW150914 is unusual.
} 

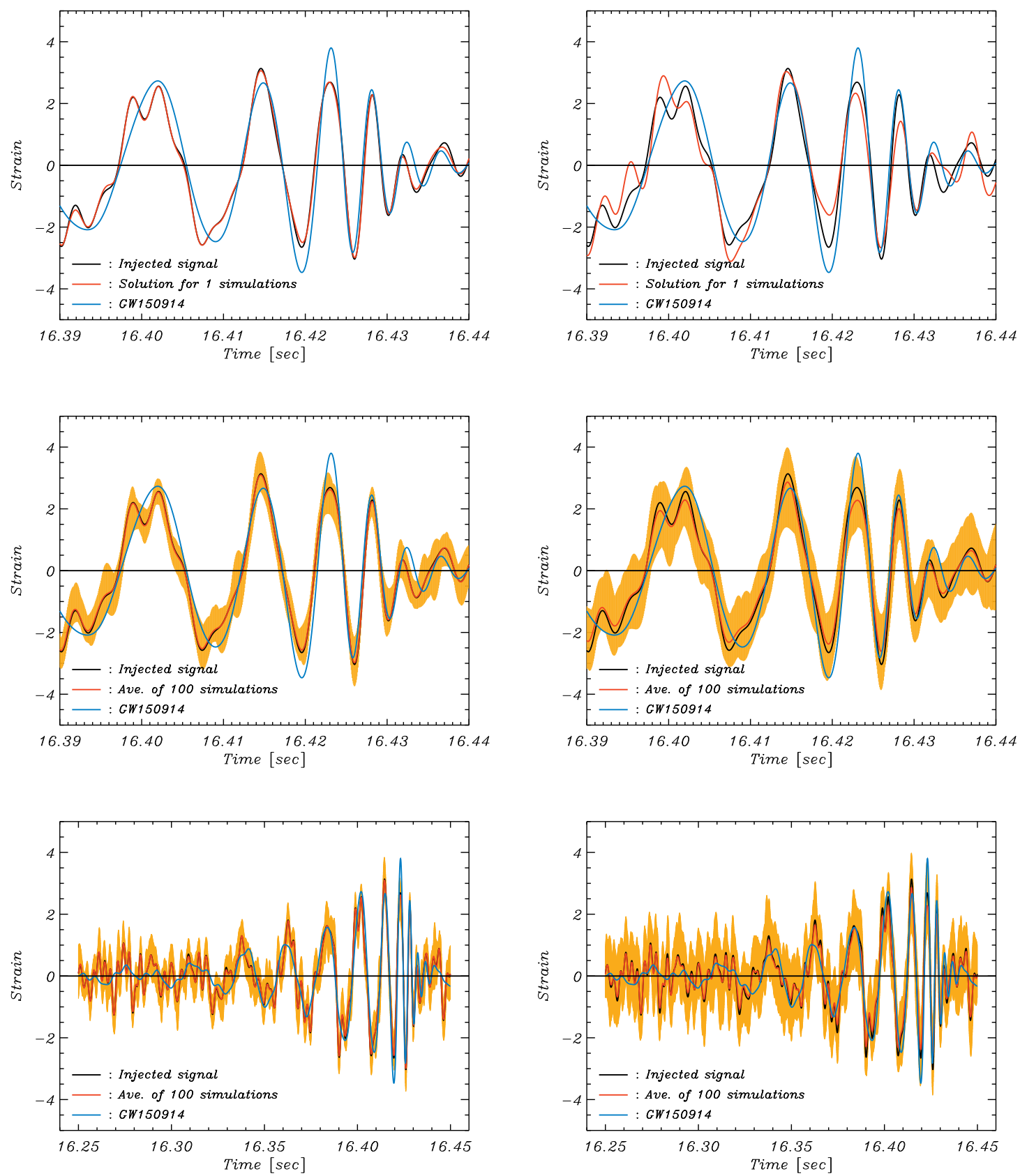

Figure 5. Results from test runs using simulated data containing the blind estimation from figure 3 and real detector noise. In the left panels the initial guess is the injected signal itself, while in the right panels the initial guess is random. Top panels: One example simulation. Middle panels: 100 simulations, including the corresponding minimal to maximal fluctuation range. Bottom panels: Same as middle but showing the full 0.2 s range.

(Livingston already matched, as explained in section 3.1):

$$
C_{i}(t)=\operatorname{Corr}\left(R_{H, t}^{t+w}, R_{\tilde{L}, t}^{t+w}\right), \quad i=1, \ldots 100
$$



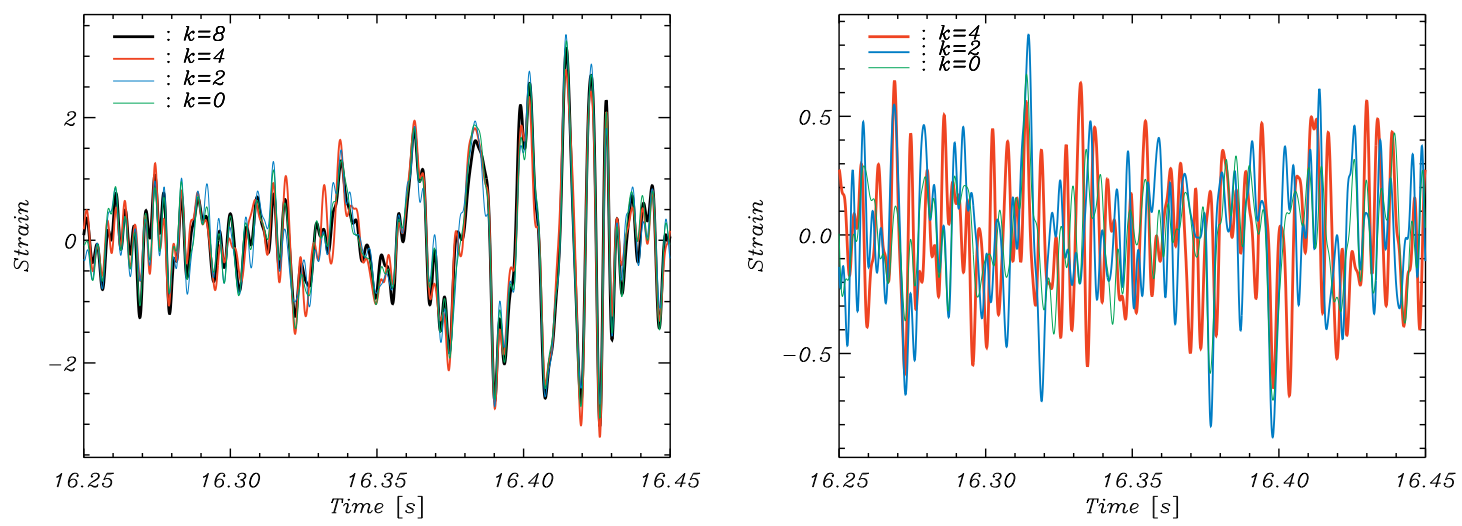

Figure 6. Test of the effect of parameter $k$ in eq. 2.6, in which we set $k=0,2,4,8$ respectively. The right panel shows the difference between these curves after subtracting the curve for $k=8$. Note that to concentrate on the effect of $k$, we use the same random initial guess for all cases.

where $X_{t_{b}}^{t_{e}}$ denotes a data stream $X(t)$, within the time range $t_{b} \leq t \leq t_{e}$ and we pick $w=0.02 \mathrm{~s}$ to be the length of the running window. For the following comparison we only consider the RMS amplitude of all correlations, $C_{i}$ :

$$
\operatorname{RMS}(t)=\sqrt{\frac{1}{N} \sum_{i=1}^{N} C_{i}^{2}(t)},
$$

and plot the resulting $\mathrm{RMS}(t)$ in figure 7 along with the absolute value of the correlation of the residuals obtained by subtracting LIGO's GW150914 template. We also show the average amplitude of the cross-correlations for comparison.

We see that our common signal estimation partially reduces the residual correlation at the precursor and main chirp regions (blue vertical lines) though the variation of the correlation still follows the red line. This might be explained by the existence of more than one "common" component, each with a potentially different amplitude ratio between the detectors, such that they cannot all be captured by a single estimation as here. This is similar to the situation in CMB science, where several Galactic foreground components such as synchrotron and thermal dust emission, each with a different spectrum, disturb our view on the background. It is not possible to remove these effects by assuming a single common foreground spectrum. With such an explanation, one might conclude that GW150914 is not the only common signal between the Hanford and Livingston detectors. The correlation for the residuals found above should be compared with the expected correlation in $0.2 \mathrm{~s}$ obtained from detector noise, which has a mean value of zero and has a standard deviation of 0.11. The consistency of this result with the observed value of 0.13 suggests that there is no compelling evidence for the existence of multiple common components. Even though this possibility would merit further investigation, it will not be considered here.

\subsection{Determination of the threshold}

The threshold used to constrain the likelihood (section 2.2) appears to be a natural choice considering the correlation coefficient between the GW150914 template and the Hanford data (about 0.87). We now justify this choice by evaluating the corresponding amplitude of the residuals. Increasing the 

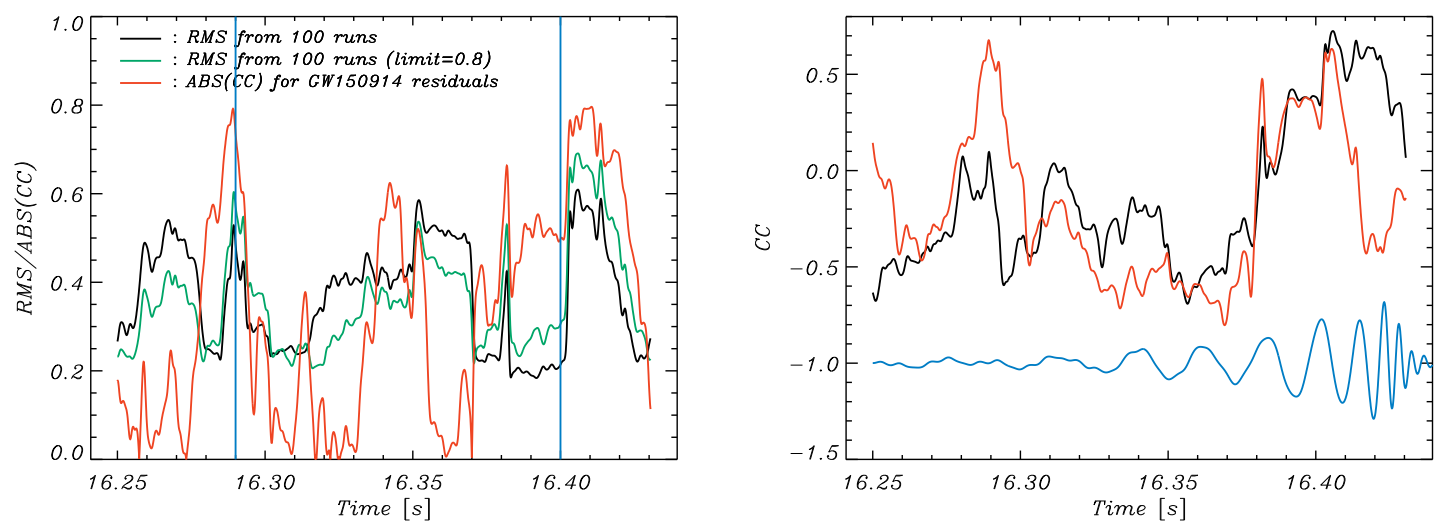

Figure 7. The residual correlation around GW150914 after subtraction of the best-fit common signals and the GW150914 template. Left panel: RMS amplitude/absolute value of correlations, including an extra test by changing the threshold from 0.9 to 0.8 . Right panel: Two examples of residual correlations from the 100 simulations calculated for a threshold of 0.9. In addition the GW150914 template (blue) is shown for guidance.

threshold leads to solutions approaching the unwanted solution shown in eq. 2.7, which in turn leads to a decrease in the residuals at one side (either Hanford or Livingston), which can be assessed by computing $\sigma_{\text {comb }}=\sigma_{R_{H}} \sigma_{R_{L}}$, where $\sigma_{X}$ is the standard deviation of quantity $X$. For each of the thresholds, 0.8, 0.9, and 0.99, we perform 100 search runs for the GW150914 data with different random initial guesses, and find $\sigma_{\text {comb }}$ to be $1.44 \times 10^{-22}, 1.35 \times 10^{-22}$, and $0.80 \times 10^{-22}$ respectively. For comparison, the same computations on pure noise segments of the same length after GW150914 result in typical values between $1.3 \times 10^{-22}$ and $1.6 \times 10^{-22}$. Thus, it is apparent that thresholds of about 0.8 to 0.9 provide a reasonable range, whereas the 0.99 is too high. The left panel fo figure 7 therefore includes results from both thresholds, 0.8 and 0.9 .

We also test the average of the RMS values for thresholds 0.8 and 0.9 shown in the left panel of figure 7 as the green and black line. These amount to 0.36 , and 0.37 , respectively. This small difference gives further support that our results are not significantly affected by the choice of the threshold.

\section{Goodness of the GW template as an estimator of the common signal}

In this section, we wish to provide a quantitative evaluation of the quality of the GW150914 template as an estimator of the common signal between Hanford and Livingston. This evaluation will be based on the likelihood of the template calculated by eq. 2.6.

First, we point out that the likelihood for the GW150914 template is 3.07 and the likelihood for the blind estimation shown in figure 3 is 3.99 . While this difference already suggests that the quality of the GW150914 template is not high, these two numbers do not tell us the probability for accepting the template. Thus, we use the following two approaches to address this question.

The first approach is to take the GW150914 template as the initial guess to start the search. If the difference between 3.07 and 3.99 is insignificant, we should see oscillations from the beginning. However, figure 8 shows a clear monotonic rise in the likelihood and a range of final oscillations that is much less that the difference between 3.99 and 3.07. This provides a qualitative indication that the difference is significant and that GW150914 is not a good estimator of the common signal. 


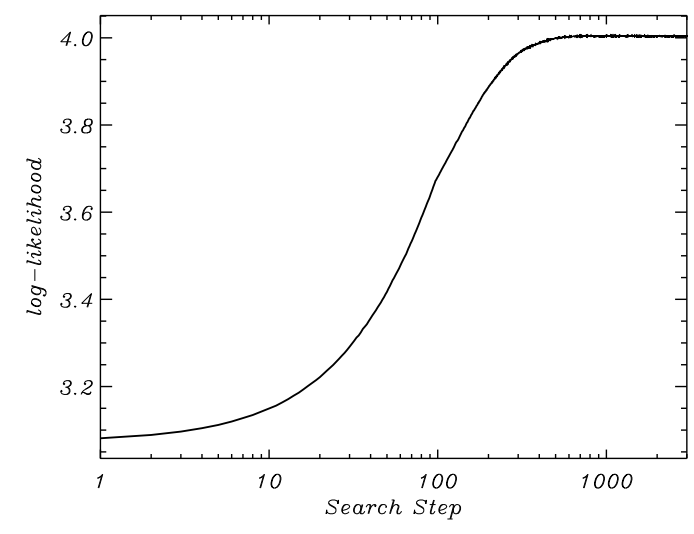

Figure 8. Evolution of the likelihood when selecting the GW150914 template as the initial guess.

The second approach is to run the search with steps, $d(t)$, that are sufficiently large to enable the solution to jump from one local maximum to another and thus prevent convergence to a single local maximum. We can then find the probability of obtaining a likelihood of 3.07 or less. This will be related to the probability that the GW150914 template should be rejected as the best common signal.

The key issue of this second approach is to ensure that the iterative steps are large enough to be able to jump between local maxima. This can be monitored by checking the likelihood of the average solution: if all steps are oscillating around only one local minimum, then the average solution will also have a high likelihood, like the one in figure 3. On the other hand, if the average solution has a very low likelihood, then the iterative steps must be jumping between multiple local maxima. Working in Fourier space, we empirically were led to adopt a step size roughly $10 \%$ of the expectation for the Fourier amplitude, and $1 \%$ of the whole size of the parameter space for the Fourier phase.

A run of $10^{6}$ iterative steps with this step size resulted in an average signal of all solutions that gives a likelihood of around 0.1. This is much lower than either 3.99 or 3.07 - sufficiently low to ensure that the iterative steps are jumping between multiple local maxima. All likelihoods are plotted in figure 9, from which we see that almost all steps are above the 3.07 line (the lowest of the horizontal, red lines). There are only 4 instances with a likelihood lower than 3.07. This suggests that the GW150914 template is preferred only with a probability of around $4 \times 10^{-6}$ compared to the best common signal found here. In figure 10 we also present a simulation using a signal composed of the best common signal of figure 3 and real noise taken from the data outside the GW150914 domain. In this case we see that the likelihood of the input signal (horizontal, red line) is consistent with the results in the region of oscillations.

Despite its appearance in figure 1 of ref. [1], the GW150914 template discussed so far is not the best-fit template. We have carried out a search to investigate the possibility that other templates are more likely according to our method. Although the dependence of template morphology on black hole masses and spins is highly degenerate in the vicinity of GW150914 [18], changes in the masses orthogonal to the chirp mass degeneracy can still affect the results.

By nature of its definition, the log-likelihood is much more sensitive than the cross-correlation to small changes in morphology. This sensitivity can be misleading since chance correlations can have a significant effect on the log-likelihood when the probability of a given template is small. Therefore, in our search, we have sought to maximize the log-likelihood by performing a Monte Carlo search over 

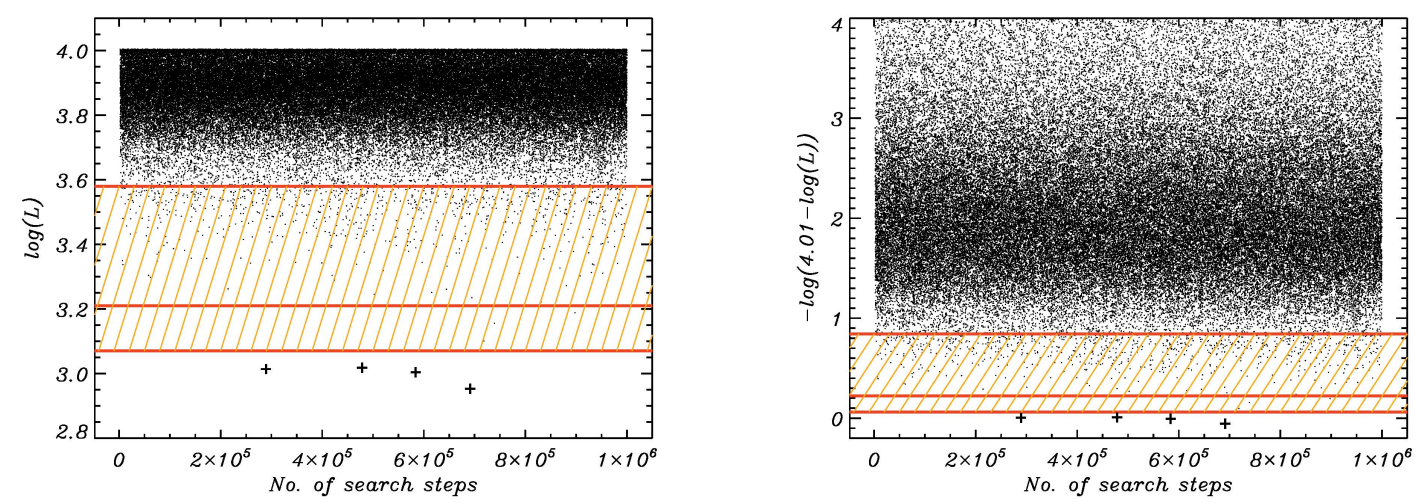

Figure 9. Likelihood as function of steps when the size of the steps are big enough to ensure jumping between local maxima. The horizontal line marks the likelihood of the GW150914 template. Both panels are identical, only that in the right panel we use the transformation $-\log (4.01-\log (L))$ to make the points close to the likelihood peak more visible. The three horizontal lines from bottom to top mark the likelihood of the GW150914 template, a large total mass template, and the maximum likelihood gravitational wave template, respectively. (See text for description.) It is clear that the GW150914 template is at a position with very low likelihood. The shaded yellow region indicates the range of likelihoods obtained from different gravitational wave templates considered here.

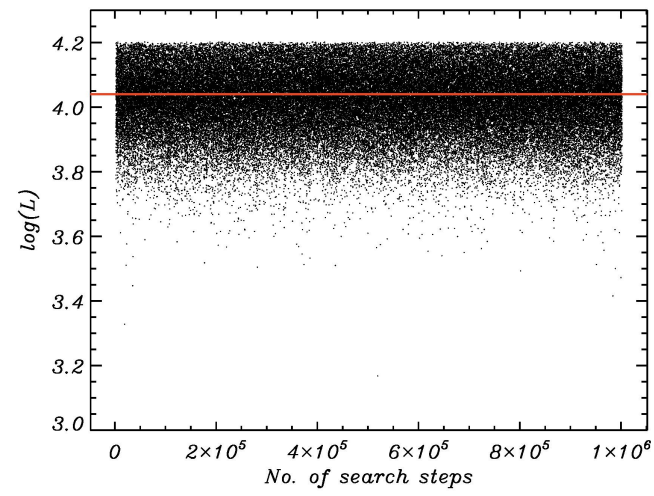

Figure 10. Same as the left panel of figure 9, but for simulation with the best common signal (see figure 3) and real detector noise.

several thousand choices of black hole templates ${ }^{11}$ with various masses and spins and have optimized the matching parameters $\Delta$ and $\tau$ to maximize the log-likelihood. A general feature of our search is that templates with component masses somewhat higher than the quoted values of 36 and 29 [14], and with anti-aligned spins, often give higher log-likelihoods. Specifically, the parameters $m_{1}=40$, $m_{2}=38, \chi_{1}=0.96$, and $\chi_{2}=-0.85\left(m_{1 / 2}\right.$ are the masses of the two black holes in units of $M_{\odot}$, and $\chi_{1 / 2}$ are their dimensionless spins) yield a maximum log-likelihood of 3.58 (shown as the highest red line in figure 9) after appropriate bandpassing, notching, and matching. This corresponds to a $p$-value of 0.008 , which is the estimated probability that the best common signal is a template

\footnotetext{
${ }^{11}$ In the following, we employ templates generated using PyCBC with SEOBNRv2 [21-24]. SEOBNRv2 was used by LIGO for analysing GW150914 [25]. A more recent version, SEOBNRv4 [26], yields the same conclusions.
} 
describing the merger of black holes.

To study further the effect of increased masses, we change the parameters of the previous template to $m_{1}=48, m_{2}=38, \chi_{1}=0.96, \chi_{2}=-0.85$, as an example, which yields a log-likelihood of 3.21 (also shown in figure 9). We note that such a template gives a high SNR in the Hanford and Livingston data streams, and thus appears to be consistent with one of LIGO's methods for finding a best-fit template.

\section{Discussion}

The precise determination of the morphology of detected gravitational waves, and particularly their deviation from theoretical templates, is important for understanding the physics of binary mergers. For example, binary mergers can be perturbed by tidal interactions with embedded stars, especially in neutron star mergers [27]. However, signal-extraction methods based on template banks are inherently biased and can overlook such features. Furthermore, template-based methods, if not handled carefully, can lead to misunderstanding or overestimation of detection significance.

In this paper we have developed a new template-free method for extraction of the common signal in two detectors. Our method is based on a likelihood approach that minimizes the Pearson cross-correlation between the residuals and maximizes the cross-correlations between the estimated common signal and the strain data. The approach is nonlinear by design and, unlike most common linear models for combining signal and noise, it gives a family of solutions differing as a consequence of the inevitable chance correlations between each member of the family and the corresponding residuals. This method makes no assumptions about the statistical properties of the noise, and cross- correlations are calculated from the data sets alone.

We have used the published $0.2 \mathrm{~s}$ Hanford and Livingston strain records for the GW150914 event [28]. We have found that the morphology of the family of common signals $A_{i}(t)$ is different from the morphology of the GW150914 template. This template deviates from the maximalminimal uncertainty range determined by the spread in the members of the family, as illustrated in figure 5. This deviation is particularly visible in the final peaks, which are incongruously high in the GW150914 template. Furthermore, one can see peculiarities in the common signals in the vicinity of the nodes, where the theoretical template is zero and the corresponding data are supposedly only noise.

In order to test the stability of our method, we have performed simulations using noise taken from Hanford and Livingston strain data well outside the GW150914 event together with injected known waveforms constructed in 100 runs of the blind search method in order to compute the fluctuation range. The comparison between injected and reconstructed waveforms, presented in figure 5, shows the stability of the method.

We are especially interested in the cross-correlation between the residuals resulting from subtraction of the common signal. Since the family of solutions $A_{i}(t)$ leads to a family of residuals, we have considered an average over 100 runs for $A(t)$ to get the moving window cross-correlations presented in figure 7 . We find a reduction of the cross-correlations in the chirp domain, detected in [6], with preservation of the precursor and the echo effects discussed therein. This suggests that the results in [6] can be explained in part as a consequence of the subtraction of the GW150914 template instead of the best common signal found in this work. ${ }^{12}$

\footnotetext{
${ }^{12}$ We would also like to mention a recent work [29] devoted to reconstruction of the common waveform from the LIGO/Virgo data. This method is based on the assumption of Gaussian noise, and more importantly, leads to a specific non-coherent signal with increasing amplitudes getting approaching to the chirp time domain. The conclusion made in [29] referring to the residual cross-correlations presented in [6] as chance correlations in the context of stationary Gaussian noise,
} 
To compare the quality of the derived waveforms with the GW150914 template, we have proposed a goodness of fit test based on the likelihood approach. The idea is to use the GW150914 template as the initial guess in the search for the best common signal. If this template is close to the best fit solution, we should see oscillations of $A_{i}(t)$ around that template. However, we have found that this is not the case. In particular, the corresponding likelihood for the initial template is 3.07, while the value for the best fit solution oscillates around 3.99. Such a gap in the likelihood strongly disfavors the GW150914 template. In addition, we have considered a large selection of templates based on other black hole parameters. The largest likelihood found was 3.58, which is significantly smaller than that of the best common signal found by the blind search algorithm. This result corresponds to a $p$-value of 0.008 . In fairness, it should be noted that this probability is a purely statistical result based on the assumption that the details of the physical description of the black hole merger are exact. It is possible that the relatively small difference between LIGO's best fit template and our best common signal is due in part to theoretical uncertainties associated with template calculations. Nevertheless, it is our view that this result emphasizes the essential importance of minimizing the cross-correlations between the residuals as well as maximizing the cross-correlations between a template and the signals from each of the two detectors.

It must be remarked, however, that the method employed requires certain properties of the signal to be true. By construction, the extracted common signal must be the only signal common to the two detectors. If more common components are present in the two detectors, our method will try to accommodate these into its best-fit common signal extraction and thereby also distort the estimation. The resulting compromises in the common signal would necessarily lead to correlations in the residuals larger than what would be expected for statistically independent noise. Furthermore, eq. 3.1 does not allow for precessing or elliptical binaries, nor does it respect higher-order modes in the gravitational waves (see also [30]). Additionally, there could be statistical and systematic errors in creating the GW-data from the detector channels [31]. In the event that such corrections were necessary, the present results would change accordingly. Evidently, similar corrections would also have to be incorporated in much of LIGO/Virgo's analysis that depends on matched filtering or the use of simplified template models.

Redundancy is a powerful tool for the extraction of weak signals in the presence of noise, and it is widely recognized as a crucial element in the detection gravitational waves. It is less obvious how this redundancy is best exploited. Template analyses can provide estimates of physical parameters given the certain knowledge that the true template is in the set considered. At their worst, template analysis can lead to a misidentification of the nature of candidate events. In our view, the only acceptable strategy is one that first finds the best common signal from the data alone using methods that are free of theoretical preconceptions and then makes the comparison to the predictions of specific physical models. We have presented one such approach here and used it to analyze the data for GW150914. Cases in which the signals are too weak to permit unbiased determination and which therefore require the use of templates for signal detection should be regarded with extreme caution: It is likely that the conclusions of such analyses will be determined by theoretical preconceptions and not by the data itself.

\section{Acknowledgments}

This research has made use of data, software and/or web, tools obtained from the LIGO/Virgo Open Science Center (https://losc.ligo.org), a service of LIGO Laboratory, the LIGO/Virgo Scientific Col-

stands in contradiction to our finding of a corresponding $p$-value of $4 \times 10^{-3}$. Moreover, considering the non-stationarity of the detector noise, it is very challenging even to define these chance cross-correlations. 
laboration and the Virgo Collaboration. This work was partially funded by the Danish National Research Foundation (DNRF) through establishment of the Discovery Center and the Villum Fonden through the Deep Space project. Hao Liu is supported by the Youth Innovation Promotion Association, CAS.

\section{References}

[1] Ligo Scientific Collaboration and Virgo Collaboration collaboration, B. P. Abbott, R. Abbott, T. D. Abbott, M. R. Abernathy, F. Acernese, K. Ackley et al., Observation of gravitational waves from a binary black hole merger, Phys. Rev. Lett. 116 (Feb, 2016) 061102.

[2] Ligo Scientific Collaboration AND Virgo Collaboration collaboration, B. P. Abbott, R. Abbott, T. D. Abbott, M. R. Abernathy, F. Acernese, K. Ackley et al., Gw151226: Observation of gravitational waves from a 22-solar-mass binary black hole coalescence, Phys. Rev. Lett. 116 (Jun, 2016) 241103.

[3] Ligo Scientific and Virgo Collaboration collaboration, B. P. Abbott, R. Abbott, T. D. Abbott, F. Acernese, K. Ackley, C. Adams et al., Gw170104: Observation of a 50-solar-mass binary black hole coalescence at redshift 0.2, Phys. Rev. Lett. 118 (Jun, 2017) 221101.

[4] Ligo Scientific Collaboration and Virgo Collaboration collaboration, B. P. Abbott, R. Abbott, T. D. Abbott, F. Acernese, K. Ackley, C. Adams et al., Gw170814: A three-detector observation of gravitational waves from a binary black hole coalescence, Phys. Rev. Lett. 119 (Oct, 2017) 141101.

[5] The LIGO Scientific Collaboration, the Virgo Collaboration, B. P. Abbott, R. Abbott, T. D. Abbott, F. Acernese et al., GW170608: Observation of a 19-solar-mass Binary Black Hole Coalescence, ArXiv e-prints (Nov., 2017), [1711.05578].

[6] J. Creswell, S. von Hausegger, A. D. Jackson, H. Liu and P. Naselsky, On the time lags of the LIGO signals, JCAP 8 (Aug., 2017) 013, [1706.04191].

[7] H. Liu and A. D. Jackson, Possible associated signal with GW150914 in the LIGO data, JCAP 10 (Oct., 2016) 014, [1609.08346].

[8] P. Naselsky, A. D. Jackson and H. Liu, Understanding the LIGO GW150914 event, JCAP 8 (Aug., 2016) 029, [1604.06211].

[9] R. A. Fisher, Frequency distribution of the values of the correlation coeffients in samples from an indefinitely large population, Biometrika 10 (1915) 507-521.

[10] R. Lynch, S. Vitale, R. Essick, E. Katsavounidis and F. Robinet, Information-theoretic approach to the gravitational-wave burst detection problem, Phys. Rev. D 95 (May, 2017) 104046.

[11] P. J. Sutton, G. Jones, S. Chatterji, P. Kalmus, I. Leonor, S. Poprocki et al., X-pipeline: an analysis package for autonomous gravitational-wave burst searches, New Journal of Physics 12 (2010) 053034.

[12] S. Klimenko, S. Mohanty, M. Rakhmanov and G. Mitselmakher, Constraint likelihood analysis for a network of gravitational wave detectors, Phys. Rev. D 72 (Dec, 2005) 122002.

[13] Ligo Scientific Collaboration and Virgo Collaboration collaboration, B. P. Abbott, R. Abbott, T. D. Abbott, M. R. Abernathy, F. Acernese, K. Ackley et al., Observing gravitational-wave transient gw150914 with minimal assumptions, Phys. Rev. D 93 (Jun, 2016) 122004.

[14] Ligo Scientific Collaboration AND Virgo Collaboration collaboration, B. P. Abbott, R. Abbott, T. D. Abbott, M. R. Abernathy, F. Acernese, K. Ackley et al., Properties of the binary black hole merger gw150914, Phys. Rev. Lett. 116 (Jun, 2016) 241102.

[15] Ligo SCIEnTific Collaboration AND Virgo Collaboration collaboration, B. P. Abbott, R. Abbott, T. D. Abbott, M. R. Abernathy, F. Acernese, K. Ackley et al., All-sky search for short gravitational-wave bursts in the first advanced ligo run, Phys. Rev. D 95 (Feb, 2017) 042003. 
[16] N. J. Cornish and T. B. Littenberg, Bayeswave: Bayesian inference for gravitational wave bursts and instrument glitches, Classical and Quantum Gravity 32 (July, 2015) 135012, [1 410 . 3835].

[17] B. Bécsy, P. Raffai, N. J. Cornish, R. Essick, J. Kanner, E. Katsavounidis et al., Parameter Estimation for Gravitational-wave Bursts with the BayesWave Pipeline, Astrophys. J. 839 (Apr., 2017) 15, [1612.02003].

[18] J. Creswell, H. Liu, A. D. Jackson, S. von Hausegger and P. Naselsky, Gravitational waveform degeneracy, To be published (2017).

[19] B. Allen, W. G. Anderson, P. R. Brady, D. A. Brown and J. D. E. Creighton, Findchirp: An algorithm for detection of gravitational waves from inspiraling compact binaries, Phys. Rev. D 85 (Jun, 2012) 122006.

[20] Ligo Scientific Collaboration and Virgo Collaboration collaboration, B. P. Abbott, R. Abbott, T. D. Abbott, M. R. Abernathy, F. Acernese, K. Ackley et al., Gw150914: First results from the search for binary black hole coalescence with advanced ligo, Phys. Rev. D 93 (Jun, 2016) 122003.

[21] T. Dal Canton, A. H. Nitz, A. P. Lundgren, A. B. Nielsen, D. A. Brown, T. Dent et al., Implementing a search for aligned-spin neutron star-black hole systems with advanced ground based gravitational wave detectors, Phys. Rev. D 90 (Oct., 2014) 082004, [1405. 6731].

[22] S. A. Usman, A. H. Nitz, I. W. Harry, C. M. Biwer, D. A. Brown, M. Cabero et al., The PyCBC search for gravitational waves from compact binary coalescence, Classical and Quantum Gravity 33 (Nov., 2016) 215004, [1508.02357].

[23] A. Nitz, I. Harry, D. Brown, C. M. Biwer, J. Willis, T. D. Canton et al., Ligo-cbc/pycbc: Post-02 release 3, 2017. 10.5281/zenodo.1058970.

[24] A. Taracchini, A. Buonanno, Y. Pan, T. Hinderer, M. Boyle, D. A. Hemberger et al., Effective-one-body model for black-hole binaries with generic mass ratios and spins, Phys. Rev. D 89 (Mar., 2014) 061502, [1311.2544].

[25] B. P. Abbott et al., Properties of Binary Black Hole Merger GW150914, Phys. Rev. Lett. 116 (2016) $241102[1602.03840]$.

[26] A. Bohé et al., Improved effective-one-body model of spinning, nonprecessing binary black holes for the era of gravitational-wave astrophysics with advanced detectors, Phys. Rev. D 95 (2017) 044028 [1611.03703].

[27] E. Annala, T. Gorda, A. Kurkela and A. Vuorinen, Gravitational-wave constraints on the neutron-star-matter Equation of State, ArXiv e-prints (Nov., 2017) , [1711. 02644 ].

[28] M. Vallisneri, J. Kanner, R. Williams, A. Weinstein and B. Stephens, The LIGO Open Science Center, in Journal of Physics Conference Series, vol. 610 of Journal of Physics Conference Series, p. 012021, May, 2015, 1410.4839 , DOI.

[29] M. A. Green and J. W. Moffat, Extraction of black hole coalescence waveforms from noisy data, ArXiv e-prints (Oct., 2017), [1711.00347].

[30] I. Harry, J. Calderón Bustillo and A. Nitz, Searching for the full symphony of black hole binary mergers, ArXiv e-prints (Sept., 2017), [1709.09181].

[31] Ligo Scientific Collaboration collaboration, B. P. Abbott, R. Abbott, T. D. Abbott, M. R. Abernathy, K. Ackley, C. Adams et al., Calibration of the advanced ligo detectors for the discovery of the binary black-hole merger gw150914, Phys. Rev. D 95 (Mar, 2017) 062003. 